aufbewahrt werden. Nach dem Auftauen muß dem Hämolysat TPN zur Analyse hinzugefügt werden.

ROBINSON (21) beschrieb kürzlich zur Erfassung von galaktosämischen Zuständen ein spezifischeres Verfahren. Dieses besteht aus einer Kombination von $\mathrm{Pa}$ pierchromatographie und Flüssigkeits-Szintillationszählung. Betr. der Einzelheiten über das Prinzip dieser Technik sei auf die Originalarbeit verwiesen.

Die von WeINBERg angegebene Methode ist sehr einfach und schnell durchzuführen, ist zuverlässig und benötigt keine teuren Reagenzien. Ein Nachteil der Isotopentechnik ist die kostspielige Apparatur zur Messung der Radioaktivität. Da diese aber weithin verfügbar ist, dürfte die von WeInBERG angegebene Technik die Methode der Wahl sein, insbesondere wenn es auf eine erste und rasche Diagnosenstellung ankommt, um die Therapie einzuleiten. Will man aber sicherstellen, daß wirklich die Blockierung der Galaktose-Glukose-Umwandlung auf einer verminderten Aktivität des Uridyl-
transferase-Systems beruht, greift man besser auf die KALCKAR-Technik in der von „Sigma“ angegebenen Modifikation zurück. Der Nachteil der KALCKAR-Methode ist ihre relative Kompliziertheit, so daß sie nur von in Enzymbestimmungen erfahrenen Laboratorien durchgeführt werden sollte. Sie ist unter keinen Umständen ein Verfahren, das man nur gelegentlich anwenden dürfte, wie die von WeINBERg angegebene IsotopenTechnik.

\section{Anmerkung bei der Korrektur}

Während der Drucklegung kam eine 1964 beschriebene Methode [E. BEUTLER, M. BALLUDA und G. N. DoNNELL: J. Lab. Clin. Med. 64, 694 (1964)] zur Erraảssung von Galaktosämikern und deren Heterozygoten zur Kenntnis des Autors. Diese Technik wurde von HSIA überprüft und beruht auf Umwandlung der Galaktose-1-P zu Glukose6-P durch Galaktose-1-P Uridyltransferase und Phosphoglukomutase im Bluthämolysat. Glukose-6-P wird anschließend durch die im Blut vorhandene Dehydrogenase oxydiert, wobei zugefügtes NADP zu NADPH reduziert wird. NADPH gibt den Wasserstoff an Methylenblau ab, das zur farblosen Form umgewarídelt wird. Die Zeit, die für

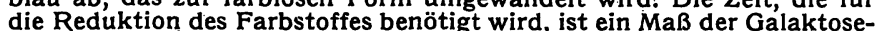
1-P Uridyltransferase Aktivität. Die Autoren fanden für die von ihnen angegeberien Bedingungen die folgenden Werte für nichtanzmische angegebenen Bedingungen die folgenden Werte fur nichtanamische Galaktosämiker: 》 3 Stḍn.

\title{
Literatur
}

1. LELOIR, L. F., The metabolism of hexosephosphates, in phosphorus metabolism, S. 67. The John Hopkins Press, Baltimore (1951). 2. Leloir, L. F., Arch. Biochem. Biophysics 33, 186 (1951). 3. KALCKAR, H. M. B. BRAGANCA und A. MUNCH-PETERSEN, Nature (London) 172,1038 (1953). - 4. SEGAL, S., A. BlaIR und Y. TOPPER, Science (Washington) 136, 150 (1962). - 5. SEGAL, S., A. BLAIR und H. Roth, Amer. J. Med. 38, 62 (1965). - 6. HANSEN, R. G., R. K. Bretthauer, J. Mayes und J. H. Nordin, Soc. exp. Biol. Med. 115, 560 (1964). - 7. Donnell, G. N., W. R. Bergren und R. S. Cleland, Pediatr. Clin. N. America 7, 315 (1960). - 8. AvigaD, G., D. Amaral, C. AsENSIO und B. L. HoRECKER, J. biol. Chemistry 237, 2736 (1962). -9. Frings, C.S. und H. L. Pardue, Analytic. Chem. 36, 2477 (1964). - 10. Raymond, A. L. und J. G. Blanco, J. biol. Chemistry 79, 649 (1928). - 11. Meranze, D. R., W. B. Likoff und G. SchNeEberg, Amer.
J. Clin. Path. 12, 261.(1942). - 12. Fiessinger, N. und A. SchrumPF C. R. Séances Soc. Biol. Filiales Associées 109, 289 (1932). - 13. TyGSTRup, N., K. WINKLER, E. Lund und H. C. ENGEll, Scand. J. Clin. Laborat. Invest. 6, 43 (1954). - 14. ANDERSON, E. P., H. M. KALCKAR, K. Kurahashi und K. J. Isselbacher, J. Laborat. Clin. Med., S. Louis 50, 469 (1957). 15. BRETTHAUER, R. K., R. G. HANSEN, G. Donnell und W. R. Bergen, Proc. nat. Acad. Sci., USA 45, 328 (1959). - 16. NORDIN, J. H., R. K. BRETTHAUER und R. C. HANSEN, Clin. chim. Acta (Amsterdam) 6, 578 (1961). = 17. KIRKMAN, H. N. und E. BYNUM, Ann. human Genetics 23, 118 (1959). - 18. ScHWARZ, V., J. Laborat. Clin. Med., S. Louis 56, 483 (1960). - 19. WEINBERG, A. N., Metabolism 10, 728 (1961). - 20. LONDON, M., J. MARYMOUNT jr. und J. Fuld, Pediatrics 33, 421 (1964). - 21. RoBInson, A., J. Exper. Med. 119, 359 (1963)

\section{Ionengehalt, Ionentransport und Membran-ATPase der Erythrocyten in Blutkonserven}

\author{
Von H. Grobecker und U. Piechowski
}

\author{
Aus dem Pharmakologischen Institut der Universität Düsseldorf (Direktor: Professor Dr. med. K. Greeff)
}

(Eingegangen am 24. September 1965)

\begin{abstract}
Der aktive Ionentransport gelagerter Erythrocyten ist in heparinisiertem oder defibriniertem Blut nach 11 Tagen, in Konserven mit ACD-Stabilisator erst nach 21 Tagen aufgehoben. - Die Aktivität der Transport-ATPase aus Erythrocytenmembranen bleibt selbst bei 90tägiger Lagerung des Stabilisatorblutes erhalten. Der aktive Ionentransport gelagerter Erythrocyten läßt sich durch Einschleusen von ATP in das Zellinnere (partielle Hämolyse mit anschließender Restitution) reaktivieren. - Unsere Untersuchungen sprechen somit dafür, $\mathrm{da} B$ der aktive Ionentransport gelagerter Erythrocyten nicht durch eine Schädigung des Carriersystems, sondern durch einen Mangel an energiereichen Substraten gestört ist.

Active ion transport in stored erythrocytes is not abolished until after 11 days in heparinised or defibrinated blood, or after 21 days when preserved with $A C D$ stabiliser. The activity of the transport ATP-ase from erythrocyte membranes is still unchanged after 90 days storage of stabilised blood.

The active ion transport in stored erythrocytes can be reactivated by introducing ATP into the cell (partial haemolysis, followed by restitution). Our studies support the conclusion that active ion transport in stored erythrocytes is not lost through damage to the carrier systẹm, but by deficiency of energy-rich substrates.
\end{abstract}

Während der Lagerung menschlicher Erythrocyten in Blutkonserven nimmt bei niedriger Außentemperatur der hohe intrazelluläre Kaliumgehalt ab und es wird Natrium aus dem Plasma aufgenommen. Zusatz von Glucose zu den Konserven verlangsamt diesen Ionen- austausch (1). W'erden die gelagerten Erythrocyten unter Zusatz von Glucose auf $37^{\circ}$ erwärmt, so findet durch aktiven Ionentransport eine Restitution des intrazellulären Ionengehaltes statt: Kalium wird in die Zelle hinein und Natrium gegen den Konzentrationsgradien- 
ten aus der Zelle "herausgepumpt" (2). Aus Untersuchungen von Gardos (3), Whittam (4) und HofFMAN (5) ist bekannt, daß Adenosintriphosphat (ATP) die unmittelbare Energiequelle für den aktiven Ionentransport an Erythrocyten darstellt und Glucose für die Synthese der energiereichen Phosphate benötigt wird. Die Vorstellung, daß der aktive Ionentransport durch die Zellmembran mit Hilfe eines energieabhängigen "Carriersystems" erfolgt $(6,7)$, fand in letzter Zeit eine weitere Grundlage durch Untersuchungen von Post und Mitarbeitern (8) sowie von Dunham und GLYNN (9), die in der Erythrocytenmembran eine durch Natrium und Kalium aktivierbare ATPase nachweisen konnten. Herzwirksame Glykoside, die den aktiven Ionentransport an intakten Erythrocyten hemmen, hemmen auch die durch Natrium und Kalium aktivierbare Membran-ATPase $(10,11)$.

In vorangehenden Untersuchungen fanden wir (12), daß Katzenerythrocyten, die einen niedrigen Kaliumgehalt haben, keine durch Kalium und Natrium stimulierbare Membran-ATPase besitzen, was dafür spricht, daß dieses Enzym für das "Carriersystem" der Erythrocyten notwendig ist. An Erythrocyten des Menschen, des Meerschweinchens und der Ratte stellten wir fest (13), daß in Parallele zur verschiedenen Empfindlichkeit des aktiven Ionentransportes gegen Strophanthin auch die aus diesen Erythrocyten isolierte Membran-ATPase durch das Glykosid verschieden stark gehemmt wird. Die Aktivierung durch Kalium und Natrium und die Hemmung durch Strophanthin sind daher - auch in den vorliegenden Untersuchungen - ein Kriterium, um die Aktivität der Transport-ATPase zu ermitteln. Nach Untersuchungen von BlosterN und Mitarbeitern (14) nimmt bei Erythrocyten nach längerer Lagerung in ACD-Stabilisator die Fähigkeit des aktiven Rücktransportes von Kalium und Natrium ab. Hierfür könnte sowohl die Verminderung des intrazellulären Gehaltes an ATP $(14,15)$ als auch eine Schädigung der für den Transport notwendigen Membran-ATPase verantwortlich sein. Wir haben deshalb in der vorliegenden Arbeit untersucht, ob die Transportleistung und die MembranATPase-Aktivität der in ACD-Ștabilișator gelagerten Erythrocyten in gleicher Weise gestört sind. Zum Vergleich wurden Untersuchungen an Erythrocyten durchgeführt, die in heparinisiertem oder defibriniertem Blut gelagert waren.

\section{Methodik}

Durch Venenpunktion gewonnenes Blut wurde defibriniert oder durch Zusatz von Heparin $(2 \mathrm{mg} / \mathrm{m} /)$ bzw. ACD-Stabilisator (1:5 $\nabla / v)$ ungerinnbar gemacht und bei $+4^{\circ}$ gelagert. Der ACDStabilisator hatte folgende Zusammensetzung: Glucose $2 \%$, Natriumcitrat 2,25\%, Citronensäure 0,66\%. Zur Bestimmung des intrazellulären Ionengehaltes wurden jeweils $1 \mathrm{~m} /$ Blut entnommen, zentrifugiert und die Erythrocyten $2 \mathrm{mal}$ mit eiskalter, isotonischer Cholinchloridlösung gewaschen. Nach Hämolyse der Zellen mit aqua bidest. wurde der Natrium- und Kaliumgehalt (bezogen auf $m A q / /$ Erythrocyten) flammenphotometrisch bestimmt und der intrazelluläre $\mathrm{Na}^{+} / \mathrm{K}^{+}$-Quotient berechnet.

Zur Bestimmung des aktiven Ionentransportes wurden aus Blutkonserven nach verschieden langer Lagerung je $15 \mathrm{ml}$ entnommen und unter Zusatz von Glucose $(2 \mathrm{mg} / \mathrm{m} /)$ bis zu 5 Stdn. bei $37^{\circ}$ inkubiert.

Die Präparation der Erythrocytenmembranen erfolgte nach der Methode von Post und Mitarbeitern (8). Zur Bestimmung der ATPase-Aktivität wurden Enzymansätze bei $37^{\circ} 1 \mathrm{Std}$. lang inkubiert. In einem Endvolumen von 1,5 ml enthielten sie 0,1-0,2 $\mathrm{m} l$ Enzymlösung, Trispuffer ( $\mathrm{pH} 7,95 ; 20 \mathrm{mMol})$, ATP ( $2 \mathrm{mMol})$, $\mathrm{Mg}^{++}(5 \mathrm{mMol}), \mathrm{Na}^{+}(132 \mathrm{mMol}), \mathrm{K}^{+}(10 \mathrm{mMol})$; die Enzymlösung wurde erst nach $10 \mathrm{~min}$. Vorinkubation zugesetzt. Die Reaktion wurde durch Zusatz von $0,1 \mathrm{ml}$ eiskalter, 60-proz. Perchlorsäure im Eisbad beendet, das gefällte Protein abzentrifugiert und im Uberstand der anorganische Phosphor nach FISKE und Subbarow (16) bestimmt. Außerdem ermittelten wir als Bezugswert den Proteingehalt der Ansätze (Biuret). In Kontrollansätzen ohne Enzymzusatz haben wir den spontanen ATP-Zerfall ermittelt und die Werte der Versuche entsprechend korrigiert. In den Enzympräparaten selbst ließ sich kein anorganischer Phosphor nachweisen. Die Einführung von ATP in die Erythrocyten erfolgte durch partielle Hämolyse und anschließende Restitution, wie sie von Gardos (3) und Hoffman und Mitarbeitern (17) angegeben wurden.

\section{Ergebnisse}

\section{Einfluß der Lagerung auf Ionengebalt und Ionentransport}

Durch Venenpunktion gewonnenes Blut gesunder, freiwilliger Spender wurde defibriniert oder mit Heparin bzw. ACD-Stabilisator versetzt, der $\mathrm{Na}^{+}$- und $\mathrm{K}^{+}$ Gehalt der Erythrocyten sofort bestimmt und das Blut 3 und 6 Tage bei $+4^{\circ}$ im Kühlschrank unter sterilen Bedingungen gelagert. Der $\mathrm{Na}^{+} / \mathrm{K}^{+}-$Quotient der Erythrocyten betrug vor der Lagerung 0,08-0,11 und war in den drei verschiedenen Konserven etwa gleich groß. Nach 3tägiger Lagerung stieg der Quotient durch Austausch der Ionen in Richtung der Konzentrationsgradienten an. Dieser passive Ionenaustausch erfolgte im heparinisierten und defibrinierten Blut wesent-

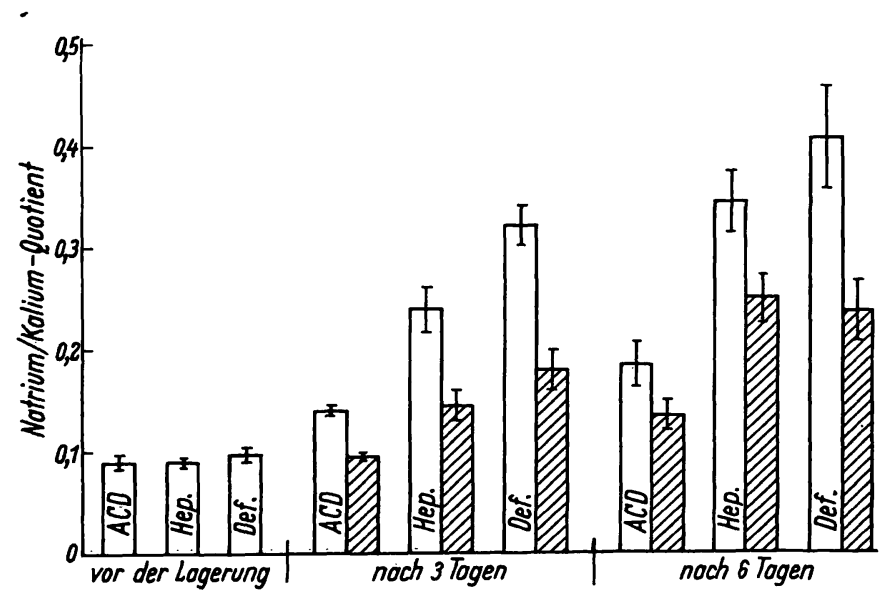

Abb. 1

Anderung des intrazellulären $\mathrm{Na}^{+} / \mathrm{K}^{+}-\mathrm{Quotienten} \mathrm{menschlicher}$ Erythrocyten nach 3- bzw. 6 tägiger Lagerung bei $+4^{\circ}$ und anschließender 5 stdg. Inkubation bei $37^{\circ}$

$A C D=$ Exytbrocyten aus Konserven mit ACD-Stabilisator;

Hep. = Erythrocyten aus heparinisiertem Blut;

Def. = Erythrocyten aus defibriniertem Blut;

Die schwarzen Säulen bedeuten die Quotienten nach 3stdg. Inkubation bei $37^{\circ}$ (aktiver Transport). Mittelwerte und mittlere Fehler der Mittelwerte von je 4 Versuchen mit verschiedenen Konserven 
Abb. 2

Intrazellulärer $\mathrm{Na}^{+} / \mathrm{K}^{+}$ Quotient menschlicher Erythrocyten nach 6-, 11bzw. 21 tägiger Lagerung bei $+4^{\circ}$ und anschließender 5 stdg. Inkubation bei $37^{\circ}$

O Defibriniertes Blut O heparinisiertes Blut $\triangle$ ACD-Stabilisator-Blut
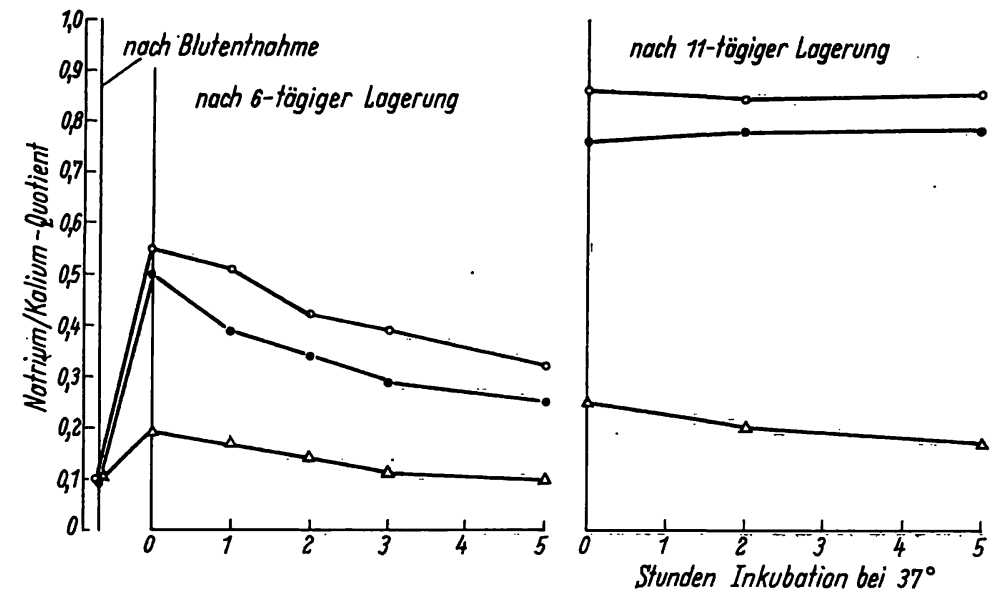

nach 21-tögiger lagerung

Tab. 1

Die Aktivität der durch $\mathrm{Na}^{+}+\mathrm{K}^{+}$aktivierbaren und durch Strophanthin hemmbaren Transport-ATPase der Erythrocytenmembran bleibt auch nach längerer Lagerung im ACD-Stabilisator erhalten Inkubationsmedium: ATP $2 \mathrm{mMol}, \mathrm{Mg}^{++} 5 \mathrm{mMol}, \mathrm{Na}+132 \mathrm{mMol}, \mathrm{K}+10 \mathrm{mMol}$, Tris $20 \mathrm{mMol} \mathrm{pH} 7,9\left(1 \mathrm{Std} .37^{\circ}\right)$

\begin{tabular}{|c|c|c|c|}
\hline \multirow{2}{*}{$\begin{array}{l}\text { Dauer der } \\
\text { Lagerung } \\
\end{array}$} & \multicolumn{3}{|c|}{ 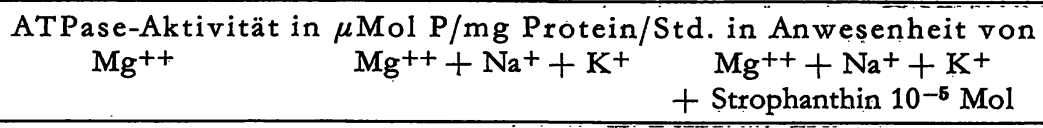 } \\
\hline & 0,28 & 0,53 & 0,27 \\
\hline 8 & 0,29 & 0,52 & 0,23 \\
\hline 21 & 0,24 & 0,49 & 0,20 \\
\hline 95 & 0,25 & 0,50 & 0,24 \\
\hline
\end{tabular}

lich schneller als im Stabilisatorblut. Noch deutlicher wurde dieser Unterschied nach Gtägiger Lagerung: in heparinisiertem bzw. defibriniertem Blut war der Quotient auf das 3-4fache, in Stabilisatorblut dagegen nur auf das Doppelte angestiegen (Abb. 1).

Blostein u. Mitarbeiter (14) fanden in ähnlichen Versuchen, daß der verzögerte passive Kationenaustausch nach Zusatz von ACD-Stabilisator durch die höhere $\mathrm{H}^{+}$-Ionenkonzentration bedingt ist. Andererseits hemmt auch Glucose, die unmittelbar vom. Stoffwechsel der Erythrocyten zum Aufbau von ATP verwendet wird, diesen passiven Ionenaustausch (1).

Unabhängig von der Art der Lagerung als defibriniertes, heparinisiertes oder ACD-Blut fand bei allen $3 \mathrm{bzw}$. 6 Tage alten Erythrocyten während einer 3stdg. Inkubation bei $37^{\circ}$ ein Anstieg des intrazellulären Kaliumgehaltes und eine Abnahme des intrazellulären Natriumgehaltes statt (Abb. 1). Dieser Abfall des $\mathrm{Na}+/ \mathrm{K}^{+}$ Quotienten erfolgte gegen das Konzentrationsgefälle und war somit ein $\mathrm{MaB}$ der aktiven Transportleistung durch die Zellmembran. Bei längerer Lagerung nahm die Fähigkeit des aktiven Rücktransportes jedoch deutlich ab. Nach 11tägiger Lagerung trat bei Erythrocyten aus heparinisiertem und defibriniertem Blut während der Inkubation bei $37^{\circ}$ kein Abfall des $\mathrm{Na}^{+} / \mathrm{K}^{+}-$Quotienten mehr ein, nach 21 tägiger Lagerung war auch bei Erythrocyten aus ACD-Konserven der aktive Ionentransport aufgehoben (Abb. 2).

Um festzustellen, ob bei gelagerten Erythrocyten durch Inkubation mit frischem Plasma der aktive Ionentransport wiederhergestellt werden kann, haben wir Erythrocyten von $A C D-K o n s e r v e n$ nach 16-21 tägiger Lagerung mit 0,9 proz. $\mathrm{NaCl}-\mathrm{Lösung}$ gewaschen und im frisch entnommenen Blutplasma des gleichen Spenders re- șuspendiert. In 5 Versuchen ließ sich keine Reaktivierung des Ionentransportes nachweisen.

Einfluß $\beta$ der Lagerung auf die Aktivität der Membran-ATPase Aus Erythrocyten der heparinisierten, defibrinierten oder ACD-Blutkonserven ließ sich eine in gleicher Weise durch Kalium und Natrium aktivierbare und durch Strophanthin hemmbare Membran-ATPase darstellen. Auch nach 10tägiger Lagerung, wenn Erythrocyten in heparinisiertem oder defibriniertem Blut nicht mehr in der Lage waren, Kalium und Natrium zu transportieren, bestand kein Unterschied der Enzymaktivität gegenübèr Erythrocyten aus ACD-Konserven. Wir haben deshalb die Aktivität der Membran-ATPase von Erythrocyten aus ACD-Konserven bis zum 95. Tag der Lagerung untersucht. In Tabelle 1 sind als Beispiel die Versuchsergebnisse mit der gleichen Blutkonserve nach 8-, 21- und 95tägiger Lagerung dargestellt: am 21. Tag, als der aktive Ionentransport bereits vollständig aufgehoben war (gleiche Konserve wie in Abb. 2), war die Aktivität der Membran-ATPase verglichen mit derjenigen der frischen Erythrocyten unverändert. Selbst nach 95 Tagen ließ sich keine Änderung der durch Kalium und Natrium akțivierbaren und durch Strophanthin hemmbaren Enzymaktivität nachweisen. Aus diesen Versuchen geht hervor, daß bei der Lagerung der Erythrocyten die Aktivität der Transport-ATPase länger . erhalten bleibt als ihre Fähigkeit des aktiven Ionentransportes. Versuche zur Wiederberstellung des aktiven Ionentransportes
durch $A T P$

Nach den vorangegangenen Versuchen war zu vermuten, daß der aktive Ionentransport gelagerter Erythro- 
Tab. 2

Wiederherstellung des aktiven Ionentransportes menschlicher Erythrocyten durch intrazelluläre Anreicherung von ATP Nach 18 bzw. 28 Tage langer Lagerung der Erythrocyten in ACD-Konserven ist der aktive Transport aufgehoben und wird durch partielle Hämolyse und anschließende Restitution unter Zusatz von $\mathrm{Mg}^{++}(148 \mu \mathrm{Mol})$ und ATP $(322 \mu \mathrm{Mol})$ reaktiviert. Inkubationsmedium: $\mathrm{Na}^{+}=147 \mathrm{mMol}, \mathrm{K}^{+}=8 \mathrm{mMol}, \mathrm{k}-$ Strophanthin $=10^{-7} \mathrm{Mol}$

\begin{tabular}{|c|c|c|c|c|}
\hline $\begin{array}{l}\text { Erythrocyten aus } \\
\text { ACD-Konserven }\end{array}$ & vor Inkubation & $\begin{array}{r}\text { Intrazellulärer } \mathrm{N} \\
\text { mit Strophanthin }\end{array}$ & $\begin{array}{l}\text { Quotient } \\
\left.\text { abation (3 Stdn. } / 37^{\circ}\right) \\
\text { ohne Strophanthin }\end{array}$ & Differenz \\
\hline \multicolumn{5}{|c|}{ nach 18tägiger Lagerung } \\
\hline unbehandelt & 0,35 & 0,35 & 0,35 & 0 \\
\hline partiell hämolysiert & 2,32 & 2,79 & 2,09 & $-0,70$ \\
\hline \multicolumn{5}{|c|}{ nach 28tägiger Lagerung } \\
\hline unbehandelt & 0,68 & 0,68 & 0,68 & 0 \\
\hline partiell hämolysiert & 2,01 & 2,46 & 1,82 & $-0,64$ \\
\hline
\end{tabular}

cyten nicht durch eine Schädigung der hierfür notwendigen Transport-ATPase, sondern durch einen Mangel an energetischem Substrat bedingt ist. BLOSTEIN und Mitarbeiter (14) sowie KLEINE und Schmitr (15) konnten nachweisen, daß der ATP-Gehalt der Erythrocyten während der Lagerung im ACD-Stabilisator kontinuierlich abnimmt. Wir führten deshalb zunächst einige Versuche durch, in denen wir 20-35 Tage gelagertes Stabilisatorblut unter Zusatz von ATP (Endkonzentration $2,5 \mathrm{mMol}$ ) 3-5 Stunden bei $37^{\circ}$ inkubierten und anschließend den intrazellulären $\mathrm{Na}+/ \mathrm{K}+$-Quotienten bestimmten. Auf diese Weise ließ sich jedoch keine Reaktivierung des Ionentransportes bei länger als 20 Tagen gelagerten Erythrocyten erzielen.

In weiteren Untersuchungen haben wir, in Anlehnung an die Methode von Gardos (3) sowie von Hofrman und Mitarbeitern (17), eine partielle Hämolyse durchgeführt mit anschließender Restitution in einem ATPhaltigen Medium. Auf diese Weise gelingt es, ATP in die Erythrocyten einzuschleusen. In Tabelle 2 sind zwei Versuchsbeispiele dargestellt, in denen die Transportleistung der mit ATP angereicherten "Ghosts" nach 18- bzw. 28tägiger Lagerung der Erythrocyten in einer ACD-Konserve ermittelt wurde. In Kontrollansätzen blieb der $\mathrm{Na}^{+} / \mathrm{K}^{+-}$-Quotient und damit der aktive Ionentransport unvorbehandelter Erythrocyten während der 3stdg. Inkubation (wie in dem Versuch der Abb. 2) unbeeinflußt. Die mit ATP angereicherten Erythrocyten waren dagegen während der $3 \mathrm{stdg}$. Inkubation in der Lage, ihren $\mathrm{Na}+/ \mathrm{K}^{+}-$Quotienten $\mathrm{zu}$ vermindern, d. h. Kalium aufzunehmen bzw. Natrium abzugeben. Der aktive Ionentransport an diesen Erythrocyten ist besonders deutlich zu erkennen, wenn man die Quotienten dieser Ansätze mit denjenigen vergleicht, in denen der aktive Ionentransport durch Strophanthin gehemmt war (Tab. 2).

\section{Diskussion}

Unsere Untersuchungen haben ergeben, daß menschliche Erythrocyten nach längerer Lagerung die Fähigkeit verlieren, ihren intrazellulären $\mathrm{K}$-Gehalt zu regenerieren. In Übereinstimmung mit anderen Autoren fanden wir, daß diese Transportleistung der Erythrocytenmembran länger erhalten bleibt, wenn das Blut unter Zusatz des ACD-Stabilisators gelagert wird. Während an Erythrocyten aus heparinisiertem oder defibriniertem Blut schon nach 11tägiger Lagerung kein aktiver Ionentransport mehr stattfindet, erlischt die Transportfähigkeit in Stabilisatorblut erst nach etwa 20 Tagen. Diese Feststellung ist wichtig, da die Lebensfähigkeit infundierter Erythrocyten aus Blutkonserven wahrscheinlich maßgeblich von der Erhaltung des aktiven Ionentransportes abhängt.

Die Ursache des geschädigten Ionentransportes ist nach unseren Versuchsergebnissen nicht eine Schädigung des für den Transport notwendigen Enzyms - der MembranATPase - sondern ein intrazellulärer Mangel an ATP. Wir fanden, daß Membranpräparate aus Erythrocyten, die bis zu 95 Tagen in ACD-Konserven gelagert waren, noch ihre volle Enzymaktivität besitzen, und konnten andererseits feststellen, daß nach Einschleusen von ATP in das Zellinnere eine Reaktivierung des aktiven Transportes möglich ist. Interessant ist, $\mathrm{da} \beta$ nach neueren Untersuchungen von SCHRIER und DOAK (18) wahrscheinlich in der Erythrocytenmembran ATP synthetisiert wird, so daß während der Lagerung der Erythrocyten möglicherweise doch eine wesentliche Schädigung der Zellmembranen stattfindet. Die Erhaltung des intrazellulären ATP-Gehaltes wird nach Untersuchungen von WOSEGIEN und Mitarbeitern (19) durch Zusatz von Purinen und Nucleosiden und nach KLEINE und SchmitT (15) durch Zusatz von anorganischem Phosphat begünstigt.

\section{Literatur}

1. Maizels, M., J. Physiology 108, 247 (1949). - 2. Harírs, J. E., J. biol. Chemistry 141, 579 (1941). - 3. GARDos, G., Acta physiol. Acad. Sci. hung. 6, 191 (1954). - 4. Whitram, R., J. Physiology 140, 479 (1958). - 5. Hoffman, J. F., Federat. Proc. 19, 127 (1960). - 6. Heinz, E., Naunyn-Schmiedebergs Arch. exp. Pathol. Pharmakol. 245, 10 (1963). - 7. WiLbRANDT, R. und Th. Rosen-
Berg, Pharmacol. Rev. 13, 110 (1961). - 8. Posr, R. L., C. R. Merrit, C.R. Kinsolving und C.D. Albright, J. biol. Chemistry 235, 1796 (1960). - 9. Dunham, E. T. und J. M. GlynN, J. Physiology 156, 274 (1961). - 10. SchatzmanN, H. J., Helvet. physiol. pharmacol. acta 11, 346 (1953). - 11. GreefF, K., Klin. Physiol. 1,340 (1963). - 12. GreefF, K., H. Grobecker und U. Piechowski, 
Naturwissenschaften 51, 42 (1964). - 13. GroBeCKER, H., U. Piechowski und K. GreefF, Med. exp. (Basel) 9, 273 (1963). 14. Blostern, R., D. Rubinstein und O. F. Denstedt, Canad. J. Biochem. Physiol. 39, 1879 (1961). - 15. Kleine, N. und H. Schmitt, Blut 8,145 (1962). - 16. Fiske, C. H. und Y. Subbarow, J. biol.
Chemistry 66, 375 (1925). - 17. Hoffman, J. F., D. C. Tosteson und R. Whittam, Nature (London) 185, 186 (1960). - 18. SChrier, S. L. und L. S. DoAK, J. Clin. Invest. 42, 756 (1963). 19. Wosegren, F., K. Dose und H. Fischer, Klin. Wschr. 40, 589 (1962).
Dr. H. Grobecker

Pharmakolog. Inst. der Universität

6 Frankfurt/Main-S 10, Ludwig-Rehn-Str. 14

\title{
Quantitative Bestimmung von $6 \beta-H y d r o x y c o r t i s o l$ unter Verwendung der Dünnschichtchromatographie mit Fluoreszenzindikator
}

\author{
Von K. Berthold und HJ. Staudinger \\ Aus dem Phjsiologiscb-Chemischen Institut der Justus-Liebig-Universität Gießen (Direktor: Prof. Dr. Hj. Staudinger)
}

(Eingegangen am 16. November 1965)

\begin{abstract}
Eine neue Methode zur Bestimmung von $6 \beta$-Hydroxycortisol im menschlichen Harn wird beschrieben. Die Methode ist spezifisch, genügend genau und richtig und als Routineverfahren geeignet. Die Ausscheidung von $6 \beta$ Hydroxycortisol bei 20 gesunden Menschen (von 20-50 Jahren) wird bestimmt. Der Normalwert ist $530 \mu \mathrm{g} /$ 24 Stdn. $\pm 140 \mu \mathrm{g}$. Nach Barbitursäuregaben sind die Ausscheidungen im allgemeinen zwar höher, sie liegen aber nicht außerhalb der Normalwertegrenzen.

A new method is described for the determination of $6 \beta$-hydroxycortisol in human urine. The method is specific and sufficiently accurate and suitable as a routine procedure. The excretion of $6 \beta$-hydroxycortisol was determined for 20 healthy adults ( $20-50$ years). The normal value was $530 \mu \mathrm{g} / 24 \mathrm{hrs}$. $\pm 140 \mu \mathrm{g}$. After the administration of barbituric acid, the excretion is generally higher, but it does not lie outside the range of normal values.
\end{abstract}

6 $\beta$-Hydroxycortisol, das erstmals 1954 von Burnstein, DorfaAan und NADEL (1), zunächst in Meerschweinchen-Harn und später auch in menschlichem Harn nachgewiesen werden konnte, wurde von FranTZ, KATZ und JAILER, sowie ULSTROM und Mitarbeitern (2-5) in erhöhten Konzentrationen im menschlichen Urin bei normaler Schwangerschaft, bei Schwangerschaftstoxikosen, bei Neugeborenen und bei der Nebennierenrindenüberfunktion gefunden. Aus Rattenharn haben $\mathrm{H}$. HeRkEN und E. SEEBER $6 \beta$-Hydroxycorticosteron isolieren können (6).

Besondere Bedeutung erhielt die Bestimmung von $6 \beta$ Hydroxycortisol im Harn, nachdem ConNey und KLUTCH (7) im Rahmen ihrer Untersuchungen zeigen konnten, daß durch Phenobarbital neben anderen mikrosomalen mischfunktionellen Oxygenasen in der Rattenleber auch die $6 \beta$-Steroid-Hydroxylase induziert wird. Setzt man voraus, daß eine Aktivierung von mikrosomalen Enzymsystemen auch in der menschlichen Leber möglich ist, so könnte die $6 \beta$-Hydroxycortisol-Ausscheidung beim Menschen ein geeigneter Parameter für die mikrosomale Leberfunktion sein. Mit der von Nishrkaze, Abraham und Staudinger angegebenen Methode zur dünnschichtchromatographischen Fraktionierüng von Corticosteroiden im Harn $(8,9)$ kann $6 \beta$-Hydroxycortisol nicht erfaßt werden. Seine relativ gute Löslichkeit in Wasser machte eine Erweiterung der von FranTZ und Mitarbeitern (4) zur Isolierung von $6 \beta$-Hydroxycortisol angegebenen Verteilungsverfahren und eine Entwicklung anderer Laufmittelsysteme für die Dünnschichtchromatographie, als sie zur Isolierung der weniger polaren Harncorticosteroide notwendig sind $(8,9)$, erforderlich.

Setzt die Einführung der Dünnschichtchromatographie zur Fraktionierung von Corticosteroiden im Harn den zeitlichen und methodischen Aufwand erheblich herab,

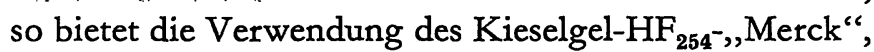
das einen Fluoreszenzindikator enthält, eine weitere methodische Vereinfachung. Die durch die $\Delta^{4}-3-K e t o-$ Gruppe des A-Ringes bedingte Löschung der Fluoreszenz auf der Dünnschichtplatte macht nach der Chromatographie eine unmittelbare Lokalisation des $6 \beta-\mathrm{Hy}-$ droxycortisols möglich. Nachfolgend wird die Methode ausführlich dargestellt, da schon geringe methodische Abweichungen die Reproduzierbarkeit der quantitativen Bestimmung in Frage stellen.

\section{Methodik \\ Extraktion}

Der 24-Stdn.-Harn soll, um Verluste an $6 \beta$-Hydroxycortisol zu vermeiden, möglichst bald nach dem Sammeln aufgearbeitet werden. Man kann ihn jedoch auch bei -10 bis $-15^{\circ}$ einfrieren, wobei der $6 \beta$-Hydroxycortisolgehalt konstant bleibt.

$1 / 20$ eines 24-Stdn.-Harns wird mit Essigsäure auf $\mathrm{pH} 4,5$ eingestellt, mit Natriumsulfat (20\% der Harnmenge) versetzt und zweimal mit dem doppelten Volumen Äthylacetat jeweils $1 / 2$ Std. geschüttelt. Die vereinigten Extrakte werden dreimal mit 0,5 $n-\mathrm{NaOH}$ in 20-proz. Natriumsulfatlösung ( $1 / 20$ des Extraktionsvolumens) und einmal mit aqua dest. ( $1 / 100$ des Extraktionsvolumens) gewaschen, durch ein Faltenfilter in einen Rundkolben filtriert und bei 37 bis $40^{\circ}$ unter Stickstoff eingedampft. Die Lösung des Rückstandes in $5 \mathrm{~m} /$ Methylenchlorid / Methanol (4:1) überführt man in ein $10 \mathrm{~m} /$-Schliffröhrchen und dampft sie bei 37 bis $40^{\circ}$ unter Stickstoff ein. Der Rückstand wird in $1 \mathrm{~m} l$ Methylenchlorid aufgenommen und mit $8 \mathrm{ml}$ methylenchloridgesättigtem Wasser etwa 3 Min. geschüttelt. Nach Zentrifugieren der entstandenen Emulsion trennt man die untere Phase ab, schüttelt den Uberstand etwa 3 Min. mit $0,5 \mathrm{~m} /$ Methylenchlorid und zentrifugiert erneut. Die wäßrige Phase wird abgehebert und zweimal mit $50 \mathrm{~m} /$ wassergesättigtem Äthylacetat etwa $10 \mathrm{Min}$. extrahiert. Die beiden Phasen werden durch Zentrifugieren entmischt. Die vereinigten Extrakte dampft man im Rundkolben bei 37 bis $40^{\circ}$ unter Stickstoff ein, überführt den Rückstand mit etwa $5 \mathrm{~m} /$ Methylenchlorid/Methanol $(4: 1)$ in ein Schliffröhrchen und verdampft das Lösungsmittel: 